\title{
On Periodic Pollaczek Waiting Time Processes
}

\author{
J.W. Cohen
}

CWI

P.O. Box 94079, 1090 GB Amsterdam, The Netherlands

January, 1994

\begin{abstract}
Present-day modelling of traffic in broadband communication requires the use of rather sophisticated stochastic processes. Although a large class of suitable stochastic processes is known in the literature, their rather complicated structure limits their use because of the laborious numerical evaluation involved. The present study concerns the so-called periodic Pollaczek processes. The characteristics of the arrival process, such as service time $\tau_{n}$ and interarrival time $\sigma_{n}$, are here periodic functions of $n$, i.e. the vector $\left(\tau_{n}, \sigma_{n}\right)$ and $\left(\tau_{n+N}, \sigma_{n+N}\right)$ have the same distribution, $N$ being the period. The sequence $\mathbf{w}_{n}, n=1,2, \ldots$, defined by $\mathbf{w}_{n+1}=\left[\mathbf{w}_{n}-\tau_{n}-\sigma_{n}\right]^{+}$

is investigated; as such the queue under consideration is a direct generalisation of the classical Pollaczek $G I / G / 1$ queue. It appears that the model is a quite flexible one, and moreover very accessible for numerical evaluation if the distributions of all the service times, or of all the interarrival times, have rational LaplaceStieltjes transforms.
\end{abstract}

AMS Subject Classification (1991): 90B22, 60K25

Keywords \& Phrases: periodic arrival processes, actual waiting times, stationary distributions

\section{INTRODUCTION}

For the classical $G I / G / 1$ waiting time model Pollaczek characterises the structure of the actual waiting time process $\mathbf{w}_{n}, n=1,2, \ldots$, by the relations

$$
\begin{aligned}
\mathbf{w}_{n+1} & =\left[\mathbf{w}_{n}+\boldsymbol{\rho}_{n}\right]^{+} \quad n=1,2, \ldots, \\
\mathbf{w}_{1} & =w_{1} \geq 0,
\end{aligned}
$$

where $\mathbf{w}_{n}$ is the actual waiting time of the $n$th arriving customer, $w_{1}$ the initial waiting time and

$$
\rho_{n}:=\tau_{n}-\sigma_{n}
$$

with $\tau_{n}$ the service time of the $n$th arriving customer, $\sigma_{n}$ the time between the $n$th and $(n+1) s t$ arrival. The $\tau_{n}, n=1,2, \ldots$, and similarly, the $\sigma_{n}, n=1,2, \ldots$, are i.i.d. nonnegative stochastic variables, and further $\left\{\tau_{n}, n=1,2, \ldots\right\}$ and $\left\{\sigma_{n}, n=1,2, \ldots\right\}$ are independent families.

A generalisation of this classical model may be formulated as follows. let

$$
\mathbf{x}_{n}, n=1,2, \ldots,
$$

be a discrete time Markov chain with a countable, irreducible state space $\mathcal{S}$ and with stationary transition probabilities. Here $\left(p_{i j}\right), i, j \in \mathcal{S}$, shall denote the one-step transition probabilities; $\mathbf{x}_{1}=x_{1}$ shall be the initial state of the $\mathbf{x}_{n}$-process.

Further, let $\rho(j), j \in \mathcal{S}$, be a set of stochastic variables with distributions $r_{j}(\cdot), j \in \mathcal{S}$, and let

$$
\rho_{n} \equiv \rho\left(\mathbf{x}_{n}\right), \quad n=1,2, \ldots,
$$


be stochastic variables defined on the $\mathbf{x}_{n}$-process, such that for all $n=1,2, \ldots$,

$$
\operatorname{Pr}\left\{\boldsymbol{\rho}_{n+1}<\rho, \mathbf{x}_{n+1}=j \mid \mathbf{x}_{m}=i_{m}, \rho_{m}=\rho\left(i_{m}\right), m=1, \ldots, n\right\}=r_{i_{n}}(\rho) p_{i_{n} j},
$$

for all $i_{n} \in \mathcal{S}$.

Define the sequence $\mathrm{w}_{n}, n=1,2, \ldots$, by

$$
\mathbf{w}_{n+1}=\left[\mathbf{w}_{n}+\rho_{n}\right]^{+}, \quad n=1,2, \ldots,
$$

for given initial $\mathbf{w}_{1}=w_{1} \geq 0$

The process $\left(\mathbf{x}_{n}, \mathbf{w}_{n}\right), n=1,2, \ldots$, as defined above will be called a Pollaczek waiting time process Obviously if we take $\rho_{n}$ to be the difference of two nonnegative stochastic variables $\tau\left(\mathbf{x}_{n}\right)$ and $\sigma\left(\mathbf{x}_{n}\right)$, so

$$
\rho_{n} \equiv \tau\left(\mathrm{x}_{n}\right)-\sigma\left(\mathrm{x}_{n}\right)
$$

then $\mathbf{w}_{n}$ may be interpreted as the actual waiting time of the $n$th arriving customer whose service time $\tau\left(\mathbf{x}_{n}\right)$ and next interarrival time $\sigma\left(\mathbf{x}_{n}\right)$ depend on the state of the $\mathbf{x}_{n}$-process.

The $\left(\mathbf{x}_{n}, \mathbf{w}_{n}\right)$-process has been introduced by ARJAS [2]. He presents a fairly detailed study, however, does not present results which are useful in actual performance studies. ASMUSSEN and THORISSON [3] study a similar model as Arjas, but with the $\mathbf{x}_{n}$-process replaced by a Markov process with a general state space. Their goal concerns the modelling of periodic queues. Indeed, the classical queueing models are not suited to model queueing situations with arrival processes, which lack a simple regenerative structure. The introduction of semi-Markov processes, see e.g. [7], [8], [17], has provided a greater flexibility in the modelling of the arrival, and also in that of the servicing process. The inherent numerical analysis is of course rather laborious, but generally within the limits of available computer facilities.

Queueing models with periodic arrival processes have been studied by several authors, see e.g. [3], $[4],[5],[6]$. The relevant studies mainly concentrate on the derivation of limit theorems and ergodicity conditions. Tangible results suited for numerical evaluation are hardly obtained. Actually, authors experience with the investigation of an $M / G / 1$ model with a periodical arrival process with rate $\lambda(\cdot)$ and $\lambda(t+\mu)=\lambda(t)$ for all $t>0, \mu$ being the period, is most disappointing indeed. Even for the very simple case with

$$
\begin{aligned}
\lambda(t) & =\lambda_{1} \quad \text { for } \quad 0 \leq t \leq \frac{1}{2} \mu, \\
& =\lambda_{2} \quad, \quad \frac{1}{2} \mu<t<\mu ;
\end{aligned}
$$

the analysis of the actual waiting time process is governed by a complicated integral equation, which seems hardly accessible for further analysis.

A more promising approach of periodical queueing processes is obtained whenever the characteristics of the queueing model are periodical functions of the number of arrivals. Such a model and actually a very general one is provided by the $\left(\mathbf{x}_{n}, \mathbf{w}_{n}\right)$-process introduced above, if we specify the state space $\mathcal{S}$ and the one-step transition probabilities as follows:

$$
\begin{aligned}
& \mathcal{S}=\{1,2, \ldots, N\}, \\
& \begin{aligned}
p_{i j} & =1 \text { for } j=i+1, i=1, \ldots, N-1, \text { and for } i=N, j=1, \\
& =0 \text { otherwise. }
\end{aligned}
\end{aligned}
$$

This special process will be called a periodic Pollaczek process. In section 2 the structure of this process is described and some notations are introduced. The ergodicity conditions are introduced, and it is assumed that they apply. These conditions follow from a general study of Loynes [9]. 
In section 3 the functional equations for the Laplace-Stieltjes transforms of the stationary distributions of the actual waiting times $\mathbf{w}_{1}, \ldots, \mathbf{w}_{N}$ are formulated for the case that the service times and the interarrival times do not all have lattice distributions. In section 4 the functional equations are derived for the case that all these distributions are lattice variables with state space the set of positive integers.

In section 5 we briefly discuss the case $M=1$, i.e. the classical Pollaczek model, as the well-known results given here are needed in the subsequent sections.

The functional equations derived in section 3 and 4 formulate a Hilbert Boundary Value Problem for a set of functions. Such boundary value problems have been extensively studied, see e.g. [10], [11] [12]. The somewhat special type of coefficients in our functional equations, which is due to the fact that they consist of the L.S.-transforms of the service time and interarrival time distributions, makes it possible to construct quite explicit solutions if the L.S.-transforms of all the service time distributions are rational functions without a need to specify those of the interarrival time distributions. This is also the case, if all the L.S.-transforms of the interarrival time distributions are rational.

In section 6 the periodical Pollaczek process is analysed for the case that all the service time distributions have a rational L.S.-transform. Section 7 discusses the case with the L.S.-tranforms of all the interarrival time distributions rational functions. Actually the results obtained in sections 6 and 7 are extensions of the $G I / K_{n} / 1$ and the $K_{m} / G / 1$ model, see [14].

The solutions obtained in sections 6 and 7 are quite explicit apart from the solution of a set of linear equations. The number of equations depends on the degrees of the polynomials in the denominators of the rational L.S.-transforms.

In section 8 some special variants of the periodic Pollaczek waiting process are shortly discussed; for a detailed analysis the reader is referred to [19]; the analysis concerns again the solution of standard Riemann Boundary Value Problems.

The analysis of the periodical Pollaczek waiting process shows that this process is quite flexible in modelling queueing processes with a rather complicated traffic structure, in particular they seem to be useful to describe models with bursty traffic. In the performance analysis of present day multiservice networks the models of classical queueing theory are frequently inadequate for modelling the actual queueing processes. This is due to the complicated character of the traffic to be processed by such systems. This complicated character stems from the demand to transport speach, data and video via the same network and to optimize such transport.

An intense world wide research activity is presently devoted to the modelling of traffic processing. The introduction of periodic Pollaczek waiting processes and its analysis is motivated by the need to incorporate bursty traffic in the modelling. Further algebraic and numerical analysis is needed to judge the usefulness of these period Pollaczek processes in actual performance analysis.

\section{The Periodic Pollaczek $G I_{N} / G_{N} / 1$ Queueing model}

With a slightly different notation we reformulate the $\left(\mathbf{x}_{n}, \mathbf{w}_{n}\right)$-process with the property (1.8) of the preceding section.

For a fixed integer $N \geq 1$ let

$$
\rho_{n}=\left(\rho_{1}^{(n)}, \ldots, \rho_{N}^{(n)}\right), \quad n=1,2, \ldots,
$$

be a sequence of i.i.d. stochastic vectors, with the components of each vector independent stochastic variables.

The sequence of vectors $\mathbf{w}_{n}, n=1,2, \ldots$,

$$
\mathbf{w}_{n}=\left(\mathbf{w}_{1}^{(n)}, \ldots, \mathbf{w}_{N}^{(n)}\right), \quad n=1,2, \ldots,
$$

is recursively defined by: for $n=1,2, \ldots$, 


$$
\begin{aligned}
& \mathbf{w}_{j}^{(n)}=\left[\mathbf{w}_{j-1}^{(n)}+\rho_{j-1}^{(n)}\right]^{+} \text {for } j=2, \ldots, N, \\
& \mathbf{w}_{1}^{(n+1)}=\left[\mathbf{w}_{N}^{(n)}+\rho_{N}^{(n)}\right]^{+}
\end{aligned}
$$

with initial value

$$
\mathbf{w}_{1}^{(1)}=w_{1} \geq 0
$$

Concerning the $\rho_{j}^{(n)}$ it will be assumed that it is the difference of two independent and positive variables,

$$
\rho_{j}^{(n)}=\tau_{j}^{(n)}-\sigma_{j}^{(n)}, \quad j=1, \ldots, N ; \quad n=1,2, \ldots
$$

The $\mathbf{w}_{n}$-process so defined will be called a periodical Pollaczek $G I_{N} / G_{N} / 1$ queueing model with period $N$.

We introduce some further notations and assumptions.

By $\tau_{j}, \sigma_{j}$ and $\rho_{j}, j=1, \ldots, N$, we shall denote stochastic variables such that $\tau_{1}, \sigma_{1}, \ldots, \tau_{N}, \sigma_{N}$, are all independent and for $n=1,2, \ldots$,

$$
\tau_{j} \sim \tau_{j}^{(n)}, \sigma_{j} \sim \sigma_{j}^{(n)}, \rho_{j} \sim \rho_{j}^{(n)}, j=1, \ldots, N
$$

It will be always assumed that: for $j=1, \ldots, N$,

i. $\quad \beta_{j}:=\mathrm{E}\left\{\boldsymbol{\tau}_{j}\right\}<\infty, \quad \alpha_{j}:=\mathrm{E}\left\{\sigma_{j}\right\}<\infty, \quad \gamma_{j}:=\mathrm{E}\left\{\rho_{j}\right\} ;$

ii. $\gamma=\beta-\alpha<0$;

where

$$
\beta:=\sum_{j=1}^{N} \beta_{j}, \quad \alpha:=\sum_{j=1}^{N} \alpha_{j}, \quad \gamma:=\sum_{j=1}^{N} \gamma_{j} .
$$

Further we define for $j=1, \ldots, N$,

$$
\begin{aligned}
& \alpha_{j}(\rho):=\mathrm{E}\left\{e^{-\rho \sigma_{j}}\right\}, \quad \beta_{j}(\rho):=\mathrm{E}\left\{e^{-\rho \tau_{j}}\right\}, \quad \operatorname{Re} \rho \geq 0, \\
& \gamma_{j}(\rho):=\alpha_{j}(-\rho) \beta_{j}(\rho), \quad \operatorname{Re} \rho=0,
\end{aligned}
$$

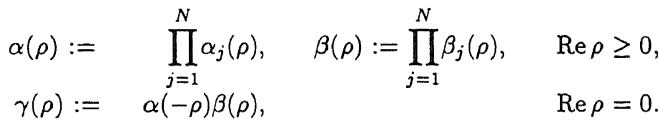

Whenever the $\alpha_{j}(\rho)$ and/or the $\beta_{j}(\rho)$ are rational functions of $\rho$ then we write: for $j=1, \ldots, N$,

$$
\alpha_{j}(\rho)=\frac{a_{1 j}(\rho)}{a_{2 j}(\rho)}, \quad \beta_{j}(\rho)=\frac{b_{1 j}(\rho)}{b_{2 j}(\rho)}, \quad \operatorname{Re} \rho \geq 0
$$

with

i. $a_{2 j}(\rho)$ a polynomial of degree $m_{j}$,

$$
b_{2 j}(\rho) \quad, \quad,, \quad,, \quad,, \quad n_{j} ;
$$

ii. $a_{1 j}(\rho)$ a polynomial of degree $<m_{j}$,

$$
b_{1 j}(\rho),, \quad,, \quad,, \quad, \quad<n_{j} ;
$$


iii. $\quad a_{1 j}(0)=a_{2 j}(0) \neq 0$,

$$
b_{1 j}(0)=b_{2 j}(0) \neq 0
$$

iv. $\quad a_{k}(\rho)=\prod_{j=1}^{N} a_{k j}(\rho) \quad b_{k}(\rho)=\prod_{j=1}^{N} b_{k j}(\rho), \quad k=1,2, \operatorname{Re} \rho \geq 0$,

$$
\mu:=\sum_{j=1}^{N} m_{j}, \quad \nu:=\sum_{j=1}^{n} n_{j}
$$

and it is assumed that the various rational functions in (2.9) are irreducible, so that

$$
\begin{array}{cccccc}
\alpha_{j}(\rho) & \text { has } & m_{j} & \text { poles } & \text { in } & \operatorname{Re} \rho<0, \\
\beta_{j}(\rho) \quad, & n_{j} & , & , & \operatorname{Re} \rho<0 ;
\end{array}
$$

these poles counted according to their multiplicities.

REMARK 2.1. Unless stated otherwise it will always be assumed that $\tau_{j}$ and $\sigma_{j}, j=1, \ldots, N$, have absolutely continuous distributions, so that, cf. [16],

$$
\begin{array}{cccccc}
\left|\alpha_{j}\left(\rho_{0}\right)\right| & =1 & \text { with } & \operatorname{Re} \rho_{0}=0 & \text { implies } & \rho_{0}=0, \\
\left|\beta_{j}\left(\rho_{0}\right)\right| & =1 & ,, & , & , & ,,
\end{array}
$$

This restriction is for the greater part of the following analysis rather inessential, but the case with all $\boldsymbol{\tau}_{j}$ and all $\boldsymbol{\sigma}_{j}$ being lattice variables requires a slightly different approach. In such a case it is more convenient to work with the generating functions of the distributions of $\boldsymbol{\tau}_{j}$ and $\sigma_{j}$, rather than using their Laplace-Stieltjes transforms, see section 4.

REMARK 2.2 . The $n$th arriving customer with $k=n \bmod N$ will be called a type " $k$ "-customer.

REMARK 2.3. Next to the vector sequence $\mathbf{w}_{n}, n=1,2, \ldots$, we introduce the vector sequence

$$
\mathbf{i}_{n}=\left(\mathbf{i}_{1}^{(n)}, \ldots, \mathbf{i}_{N}^{(n)}\right), \quad n=1,2, \ldots
$$

with

$$
\mathbf{i}_{j}^{(n)}:=-\left[\mathbf{w}_{j}^{(n)}+\boldsymbol{\rho}_{j}^{(n)}\right]^{-}, \quad j=1, \ldots, N .
$$

Obviously with probability one

$$
\mathbf{i}_{j}^{(n)} \geq 0
$$

\section{Derivation of the functional equations}

From the definition of the $\mathbf{w}_{n}$-process in the previous secton it follows that the successive epochs of the sequence

$$
\mathbf{w}_{1}^{(n)}, n=1,2, \ldots, \text { with } w_{1}=0
$$

at which $\mathbf{w}_{1}^{(n)}=0$ are regeneration points of the $\mathbf{w}_{n}$-process. Note, however, that if $\tau_{N}>\sigma_{N}$ with probability one then the sequence does not have such epochs. But the assumption (2.7)ii implies that at least one $h \in\{1, \ldots N\}$ exists for which the sequence $\mathbf{w}_{h}^{(n+h)}, n=1,2, \ldots$, does have epochs at which $\mathbf{w}_{h}^{(m)}=0$. Therefore the generality of the analysis is not restricted by assuming that $h=1$, because the arrival epoch of every type $h$-customer may be taken as the starting point of the arrival 
process. So we may and do assume that (3.1) possesses epochs at which $\mathbf{w}_{1}^{(n)}=0$.

Define

$$
\mathbf{n}_{1}:=\min _{n=1,2, \ldots}\left\{n: \mathbf{w}_{1}^{(n+1)}=0 \mid \mathbf{w}_{1}=0\right\}
$$

so that $\mathbf{n}_{1} N$ is the number of customers served in a type 1-busy period, such a period being defined as the time interval between the successsive arrival epochs of two type 1-customers with zero waiting time. Note: $\mathbf{n}_{1}$ is also the number of type $j$-customers served in a type 1-busy period.

Obviously, the $\mathbf{w}_{1}^{(n)}$-process is a random walk on $[0, \infty)$ with the zero state reflecting. It is readily seen that its drift is negative since $\gamma<0$, cf. $(2.7)$ ii, so the $\mathbf{w}_{1}^{(n)}$-process is positive recurrent, cf. [9]. Its state space is not a subset of $[0, \infty)$ if at least one of the distributions of $\tau_{j}$ or $\sigma_{j}$ is absolutely continuous. It is readily seen that the same conclusions hold for each of the sequences

$$
\mathbf{w}_{j}^{(n)}, n=1,2, \ldots, \text { with } j=1,2, \ldots, N .
$$

It follows that the $\mathbf{w}_{j}^{(n)}$-squences converge in distribution for $n \rightarrow \infty$ for every $j \in N$. Denote by $\mathbf{w}_{j}$ a stochastic variable with distribution the limiting distribution of the $\mathbf{w}_{j}^{(n)}$-sequence. It is readily shown that the sequence $\mathbf{i}_{j}^{(n)}, n=1,2, \ldots$, cf. (2.13), also converges in distribution for $n \rightarrow \infty$; denote by $\mathbf{i}_{j}$ a stochastic variable with distribution this limiting distribution. It then follows from (2.3), (2.6) and (2.13): for $j=2, \ldots, N$,

$$
\begin{array}{ll}
\mathbf{w}_{j} \sim\left[\mathbf{w}_{j-1}+\rho_{j-1}\right]^{+}, & \mathbf{i}_{j-1} \sim-\left[\mathbf{w}_{j-1}+\rho_{j-1}\right]^{-}, \\
\mathbf{w}_{1} \sim\left[\mathbf{w}_{N}+\rho_{N}\right]^{+}, & \mathbf{i}_{1} \sim-\left[\mathbf{w}_{N}+\rho_{N}\right]^{-} .
\end{array}
$$

From the identity: for real $x$

$$
\mathrm{e}^{-\rho x}+1=\mathrm{e}^{-\rho[x]^{+}}+\mathrm{e}^{-\rho[x]^{-}},
$$

we obtain from (2.3) and (3.4)

$$
\begin{aligned}
& \mathrm{e}^{-\rho \mathbf{w}_{j}^{(n+1)}}=\mathrm{e}^{-\rho\left(\mathbf{w}_{j-1}^{(n)}+\rho_{j-1}^{(n)}\right)}+1-\mathrm{e}^{\rho \mathbf{i}_{j-1}^{(n)}}, \quad j=2, \ldots, N, \\
& \mathrm{e}^{-\rho \mathbf{w}_{1}^{(n+1)}}=\mathrm{e}^{-\rho\left(\mathbf{w}_{N}^{(n)}+\rho_{N}^{(n)}\right)}+1-\mathrm{e}^{\rho \mathbf{i}_{N}^{(n)}} .
\end{aligned}
$$

Hence by taking expections in (3.5) and by noting that $\mathrm{w}_{j}^{(n)}$ and $\rho_{j}^{(n)}$ are independent, cf. section 2, it follows, cf. (2.8): for $\operatorname{Re} \rho=0 ; j=2, \ldots, N$,

i. $\quad \mathrm{E}\left\{\mathrm{e}^{-\rho \mathbf{w}_{j}}\right\}-\gamma_{j-1}(\rho) \mathrm{E}\left\{\mathrm{e}^{-\rho \mathbf{w}_{j-1}}\right\}=-\rho \mathrm{E}\left\{\mathbf{i}_{j-1}\right\} \frac{1-\mathrm{E}\left\{\mathrm{e}^{\rho \mathbf{i}_{j-1}}\right\}}{-\rho \mathrm{E}\left\{\mathbf{i}_{j-1}\right\}} ;$

ii. $\quad \mathrm{E}\left\{\mathrm{e}^{-\rho \mathbf{w}_{1}}\right\}-\gamma_{N}(\rho) \mathrm{E}\left\{\mathrm{e}^{-\rho \mathbf{w}_{N}}\right\}=-\rho \mathrm{E}\left\{\mathbf{i}_{N}\right\} \frac{1-\mathrm{E}\left\{\mathrm{e}^{\rho \mathbf{i}_{N}}\right\}}{-\rho \mathrm{E}\left\{\mathbf{i}_{N}\right\}}$.

REMARK 3.1. Note that if at least one of the distributions of $\tau_{j}$ or $\sigma_{j}, j=1, \ldots, N$, is absolutely continuous then all $\mathbf{w}_{j}$ and $\mathbf{i}_{j}$ are nonlattice variables. Further it is well known that

$$
\frac{1-\mathrm{E}\left\{\mathrm{e}^{\rho \mathbf{i}_{j}}\right\}}{-\rho \mathrm{E}\left\{\mathbf{i}_{j}\right\}},
$$

is the Laplace-Stieltjes transform of an absolutely continuous distribution with support $(-\infty, 0)$; the ergodicity of the $\mathbf{w}_{j}^{(n)}$-sequence implies that $\mathrm{E}\left\{\mathbf{i}_{j}\right\}$ is finite. 
Put for $j=1, \ldots, N$,

$$
\begin{array}{ll}
\Phi_{j}(\rho):=\mathrm{E}\left\{\mathrm{e}^{-\rho \mathbf{w}_{j}}\right\}, & \operatorname{Re} \rho \geq 0, \\
\Psi_{j}(\rho):=\frac{\mathrm{E}\left\{\mathbf{i}_{j}\right\}}{\alpha-\beta} \frac{1-\mathrm{E}\left\{\mathrm{e}^{\rho \mathbf{i}_{j}}\right\}}{-\rho \mathrm{E}\left\{\mathbf{i}_{j}\right\}}, \text { if } \mathrm{E}\left\{\mathbf{i}_{j}\right\}>0, & \operatorname{Re} \rho \leq 0,
\end{array}
$$


i. $\Phi_{j}(\rho)$ is regular for $\operatorname{Re} \rho>0$, continuous for $\operatorname{Re} \rho \geq 0$,

$$
\begin{aligned}
& \Phi_{j}(\rho)=1 \text { for } \rho=0 \\
& \Phi_{j}(\rho)=O(1) \text { for }|\rho| \rightarrow \infty,|\arg \rho| \leq \frac{1}{2} \pi
\end{aligned}
$$

ii. $\Psi_{j}(\rho)$ is regular for $\operatorname{Re} \rho<0$, continuous for $\operatorname{Re} \rho \leq 0$;

$$
\begin{aligned}
& \Psi_{j}(\rho)=\frac{E\left\{i_{j}\right\}}{\alpha-\beta} \text { for } \rho=0, \\
& \Psi_{j}(\rho)=O\left(\frac{1}{|\rho|}\right) \text { for }|\rho| \rightarrow \infty, \frac{1}{2} \pi \leq \arg \rho \leq 1 \frac{1}{2} \pi .
\end{aligned}
$$

The set of equations (3.13) for the unknown vectors $\Phi(\rho)$ and $\Psi(\rho)$, and similarly the equivalent set (3.10), formulate together with the conditions (3.15) a homogeneous Hilbert Boundary Value Problem for the $2 N$ unknown components of $\Phi(\rho)$ and $\Psi(\rho)$, with the imaginary axis $\operatorname{Re} \rho=0$ the line of discontinuity, cf. [10]. p. 384, see also [11], [12]. Actually, the functional equation (3.13) differs slightly from that discussed in [10] because the line of discontinuity is not a closed contour bounding a finite domain.

The construction of explicit solutions of these types of boundary value problem is generally hardly possible except in the three cases:

i. all $\beta_{j}(\rho)$ are rational functions,

ii. $\quad, \alpha_{j}(\rho) \quad " \quad, \quad$,

iii. for a subset of $N$ all $\beta_{j}(\rho)$ are rational

and for the complementary subset all $\alpha_{j}(\rho)$

are rational.

In the context of Queueing Theory the Hilbert Boundary Value Problem formulated above have been studied in a slightly more general form by Miller [13]. In his approach it is shown that the matrix $I-\Gamma(\rho) P$ can be written as the product of two matrices, one with all its elements regular for $\operatorname{Re} \rho<0$, the other with its elements regular for $\operatorname{Re} \rho>0$. The existence of such a factorisation is also shown in [10], however, it leads to a rather formal solution of the problem. The cases, $i$ and ii mentioned above will be analysed in the next section which quickly leads to results accessible for numerical evaluation.

REMARK 3.2. It is readily verified that for $\rho=0$ the relation (3.10) is an identity; note that $\Gamma(0)$ is the identity matrix and $\Phi_{j}(0)=1, j=1, \ldots, N$. We next consider the relation (3.10) for $\operatorname{Re} \rho=0$, $\rho \rightarrow 0$.

Because of (2.7) we have for $\operatorname{Re} \rho=0, \rho \rightarrow 0$,

$$
\begin{aligned}
\Gamma_{i j}(\rho) & =1-\rho \gamma_{j}+o(\rho) & \text { for } & i=j, \\
& =0 & , & i \neq j,
\end{aligned}
$$

so

$$
\Gamma(\rho)=I+\rho \frac{\mathrm{d}}{\mathrm{d} \rho} \Gamma(\rho)+o(\rho) I .
$$

Since $P$ has an inverse we have from (3.10) for $\operatorname{Re} \rho=0$,

$$
\Phi(\rho)\left[P^{-1}-\Gamma(\rho)\right]=(\beta-\alpha) \rho \Psi(\rho),
$$

and so by using (3.17) for $\operatorname{Re} \rho=0, \rho \rightarrow 0$, 


$$
\Phi(\rho)\left[P^{-1}-I-\rho \frac{\mathrm{d}}{\mathrm{d} \rho} \Gamma(\rho)+o(\rho) I\right]=(\beta-\alpha) \rho \Psi(\rho) .
$$

Multiply (3.18) on the right by $1^{T}$, the transposed of the unit vector $(1, \ldots, 1)$, then since

$$
\left[P^{-1}-I\right] 1^{T}=(0, \ldots, 0),
$$

we obtain for $\operatorname{Re} \rho=0, \rho \rightarrow 0$,

$$
\left[-\left.\Phi(\rho) \frac{\mathrm{d}}{\mathrm{d} \rho} \Gamma(\rho)\right|_{\rho=0}+o(1) I\right] 1^{T}=(\beta-\alpha) \Psi(\rho) 1^{T},
$$

Since, cf. (3.8) and (3.15),

$$
\Phi(0)=(1, \ldots, 1), \quad \Psi(0)=\frac{1}{\alpha-\beta}\left(\mathrm{E}\left\{\mathbf{i}_{1}\right\}, \ldots, \mathrm{E}\left\{\mathbf{i}_{N}\right\}\right),
$$

we have from (3.16) and (3.19) by letting $\rho \rightarrow 0$ that

$$
\alpha-\beta=\sum_{j=1}^{N} \gamma_{j}=\sum_{j=1}^{N} \mathrm{E}\left\{\mathbf{i}_{j}\right\}=\sum_{j=1}^{N}-\mathrm{E}\left\{\left[\mathbf{w}_{j}+\rho_{j}\right]^{-}\right\} .
$$

The relation (3.20) shows that the average total idle time during a 1-cycle, i.e. the time between successive arrivals of type 1 -customers is equal to $\alpha-\beta$; it is readily seen that the same result applies for a $j$-cycle; a $j$-cycle being defined analogously as a 1 -cycle.

\section{The functional EQUations For the LatTice CaSE.}

In the derivations of the preceding section we have used the Laplace-Stieltjes transforms of the various distributions involved. When, however, the state space of all $\tau_{j}$ and $\sigma_{j}, j=1, \ldots, N$, is the set of positive integers it is preferable to use generating functions instead of Laplace-Stieltjes transforms. In this section we shall derive the functional equation for the case of lattice variables. Exactly the same notation will be used but $p$ shall stand for the variable of the various generating functions. So we have for $j=1, \ldots, N$,

$$
\begin{aligned}
& \alpha_{j}(p):=\mathrm{E}\left\{p^{\sigma_{2}}\right\}, \quad \beta_{j}(p):=\mathrm{E}\left\{p^{\tau_{2}}\right\}, \quad|p| \leq 1, \\
& \gamma_{j}(p):=\alpha_{j}\left(p^{-1}\right) \beta_{j}(p), \quad \gamma(p):=\prod_{j=1}^{N} \gamma_{j}(p), \quad|p|=1,
\end{aligned}
$$

and if $\alpha_{j}(p)$ or $\beta_{j}(p)$ is a rational function of $p$ then

$$
\alpha_{j}(p)=\frac{a_{1 j}(p)}{a_{2 j}(p)}, \quad \beta_{j}(p)=\frac{b_{1 j}(p)}{b_{2 j}(p)}, \quad|p| \leq 1,
$$

with

$$
\begin{aligned}
& \text { i. } a_{2 j}(p) \text { a polynomial in } p \text { with degree } m_{j} \text {, } \\
& b_{2 j}(p) \quad, \quad \quad,, \quad \quad,, \quad p \quad,, \quad, \quad, \quad n_{j} \text {; } \\
& \text { ii. } a_{1 j}(p),, \quad, \quad,, \quad p \quad, \quad, \quad, \quad<m_{j} \text {, } \\
& b_{1 j}(p) \quad, \quad,, \quad,, \quad p \quad,, \quad,, \quad<n_{j} ;
\end{aligned}
$$

iii. $a_{1 j}(0)=a_{2 j}(0) \neq 0$, 


$$
b_{1 j}(0)=b_{2 j}(0) \neq 0
$$

iv. $a_{2 j}(p)$ has $m_{j}$ poles in $|p|>1$,

$$
b_{2 j}(p) \quad, \quad n_{j} \quad,, \quad,, \quad|p|>1 .
$$

It will always be assumed that $a_{1 j}(p)$ and $a_{2 j}(p)$ have no common factors, similarly for $b_{1 j}(p)$ and $b_{2 j}(p)$ and that the $\boldsymbol{\tau}_{j}$ and $\sigma_{j}$ are aperiodic lattice variables, i.e.

$$
\begin{aligned}
& \left|\alpha_{j}(p)\right|=1 \text { for } a \text { with }|p|=1 \text { implies } p=1 \text {, } \\
& \left|\beta_{j}(p)\right|=1 \quad, \quad,, \quad p \quad,, \quad|p|=1 \quad,, \quad p=1 \text {. }
\end{aligned}
$$

Again it is assumed that the sequences $\mathbf{w}_{j}^{(n)}, n=1,2, \ldots$, converge in distribution for $n \rightarrow \infty$. The variables $\mathbf{w}_{j}, \mathbf{i}_{j}, j=1, \ldots, N$, are introduced as in the previous section.

We start from the identity: for real $y$,

$$
p^{[y]^{+}}+p^{[y]^{-}}=p^{y}+1
$$

Hence

$$
\begin{aligned}
& p^{\mathbf{w}_{j}}=p^{\mathbf{w}_{j-1}+\boldsymbol{\rho}_{j-1}}+1-p^{-\mathbf{i}_{j-1}}, \quad j=2, \ldots, N, \\
& p^{\mathbf{w}_{1}}=p^{\mathbf{w}_{N}+\boldsymbol{\rho}_{N}+1-p^{-\mathbf{i}_{N}} .}
\end{aligned}
$$

So for $|p|=1, j=2, \ldots, N$,

$$
\begin{aligned}
& \mathrm{E}\left\{p^{\mathbf{w}_{j}}\right\}-\gamma_{j-1}(p) \mathrm{E}\left\{p^{\mathbf{w}_{j-1}}\right\}=-\left(1-\frac{1}{p}\right) \mathrm{E}\left\{\mathbf{i}_{j-1}\right\} \frac{1-\mathrm{E}\left\{p^{-\mathbf{i}_{j-1}}\right\}}{-\left(1-\frac{1}{p}\right) \mathrm{E}\left\{\mathbf{i}_{j-1}\right\}}, \\
& \mathrm{E}\left\{p^{\mathbf{w}_{1}}\right\}-\gamma_{N}(p) \mathrm{E}\left\{p^{\mathbf{w}_{N}}\right\}=-\left(1-\frac{1}{p}\right) \mathrm{E}\left\{\mathbf{i}_{N}\right\} \frac{1-\mathrm{E}\left\{p^{-\mathbf{i}_{N}}\right\}}{-\left(1-\frac{1}{p}\right) \mathrm{E}\left\{\mathbf{i}_{N}\right\}} .
\end{aligned}
$$

Put for $j=1, \ldots, N$,

$$
\begin{array}{ll}
\Phi_{j}(p):=\mathrm{E}\left\{p^{\mathbf{w}_{j}}\right\}, & |p| \leq 1, \\
\Psi_{j}(p):=\frac{\mathrm{E}\left\{\mathbf{i}_{j}\right\}}{\alpha-\beta} \frac{1-\mathrm{E}\left\{p^{\mathbf{i}_{j}}\right\}}{-\left(1-\frac{1}{p}\right) \mathrm{E}\left\{\mathrm{i}_{j}\right\}}, & |p| \geq 1,
\end{array}
$$

and introduce the vectors and matrices

$$
\begin{aligned}
\Phi(p) & :=\left(\Phi_{1}(p), \ldots, \Phi_{N}(p)\right) \\
\Psi(p) & :=\left(\Psi_{1}(p), \ldots, \Psi_{N}(p)\right), \\
\Gamma_{i j}(p) & :=\gamma_{j}(p) \delta_{i j}, \quad i, j \in\{1, \ldots, N\} .
\end{aligned}
$$

The relations (4.6) may now be rewritten as: for $|p|=1$,

$$
\Phi(p)[I-\Gamma(p) P]=(\beta-\alpha)\left(1-p^{-1}\right) \Psi(p) P .
$$

From (4.9) we obtain as before: for $|p|=1, p \neq 1$,

$$
\Phi(p)=(\beta-\alpha)\left(1-\frac{1}{p}\right) P[I-\Gamma(p) P]^{-1} .
$$


From the definition of $\Phi_{j}(p)$ and $\Psi_{j}(p), j=1, \ldots, N$, it follows that

i. $\Phi_{j}(p)$ is regular for $|p|<1$, continuous for $|p| \leq 1$,

$$
\Phi_{j}(1)=1
$$

ii. $\Psi_{j}(p)$ is regular for $|p|>1$, continuous for $|p| \geq 1$,

$$
\begin{aligned}
& \Psi_{j}(1)=\frac{E\left\{i_{j}\right\}}{\alpha-\beta}, \\
& \Psi_{j}(p)=O(1) \text { for }|p| \rightarrow \infty .
\end{aligned}
$$

The relation (4.9) for the vectors $\Phi(p)$ and $\Psi(p)$, and similarly the equivalent relation (4.10), formulates together with the condition (4.11) a homogeneous Hilbert Boundary Value Problem with the unit circle $|p|=1$ the contour of discontinuity, cf. [10] p. 384 .

5. SOME REMARKS ON THE CASE $N=1$

For the case $N=1$ the relation (3.10) becomes: for $\operatorname{Re} \rho=0$,

$$
\mathrm{E}\left\{\mathrm{e}^{-\rho \mathbf{w}}\right\} \frac{1-\gamma(\rho)}{(\beta-\alpha) \rho}=\frac{\mathrm{E}\{\mathbf{i}\}}{\alpha-\beta} \frac{1-\mathrm{E}\left\{\mathrm{e}^{\rho \mathbf{i}}\right\}}{-\rho \mathrm{E}\{\mathbf{i}\}},
$$

where we have written

$w$ for $w_{1}$ and $i$ for $i_{1}$.

By taking $\rho=0$ in (5.1) it follows, cf. also (3.20),

$$
\mathrm{E}\{\mathrm{i}\}=\alpha-\beta .
$$

Let $\mathbf{v}$ be a positive stochastic variable with distribution

$$
\begin{aligned}
\operatorname{Pr}\{\mathbf{v}<v\} & =\frac{1}{\mathrm{E}\{\mathbf{i}\}} \int_{0}^{v} \operatorname{Pr}\{\mathbf{i} \geq u\} \mathrm{d} u, & & v>0, \\
& =0, & & v \leq 0,
\end{aligned}
$$

then

$$
\mathrm{E}\left\{\mathrm{e}^{-\rho \mathbf{v}}\right\}=\frac{1-\mathrm{E}\left\{\mathrm{e}^{-\rho \mathbf{i}}\right\}}{\rho \mathrm{E}\{\mathbf{i}\}}, \operatorname{Re} \rho \geq 0 .
$$

Hence, it follows from $(5.1),(5.2)$ and (5.4), that: for $\operatorname{Re} \rho=0$,

$$
\mathrm{E}\left\{\mathrm{e}^{-\rho \mathbf{w}}\right\} \frac{1-\gamma(\rho)}{(\beta-\alpha) \rho}=\mathrm{E}\left\{\mathrm{e}^{\rho \mathbf{v}}\right\}
$$

The present case is actually the classical $G I / G / 1$ model with $\alpha(\rho)$ and $\beta(\rho)$ the Laplace-Stieltjes transforms of the interarrival time and of the service time distribution, with $w$ a stochastic variable with distribution the stationary distribution of the actual waiting time process and $i$ the idle time in a busy cycle, cf. [14], [15]. From the results of Queueing Theory of the $G I / G / 1$ model, or from those of Fluctuation Theory, cf. [14], II.5 and I.6.6, it follows that there exists a unique pair of functions 
$K_{-}(\rho)$ and $K_{+}(\rho)$ such that

i. $K_{-}(\rho)$ is regular for $\operatorname{Re} \rho>0$, continuous for $\operatorname{Re} \rho \geq 0$,

$K_{-}(0)=1, K_{-}(\rho)=O(1)$ for $|\rho| \rightarrow \infty, \quad|\arg \rho| \leq \frac{\pi}{2}$,

$K_{-}(\rho)$ has no zeros in $\operatorname{Re} \rho \geq 0$,

ii. $K_{+}(\rho)$ is regular for $\operatorname{Re} \rho<0$, continuous for $\operatorname{Re} \rho \leq 0$,

$K_{+}(0)=1, K_{+}(\rho)=O(-\log |\rho|)$ for $|\rho| \rightarrow \infty, \frac{1}{2} \pi \leq \arg \rho \leq \frac{3}{2} \pi$,

iii. $\quad \mathrm{e}^{K_{+}(\rho)-K_{-}(\rho)}=\frac{1-\gamma(\rho)}{(\beta-\alpha) \rho}, \quad \operatorname{Re} \rho=0$,

provided $\beta-\alpha<0$, and

$$
\sum_{j=1}^{N}\left(\tau_{j}-\sigma_{j}\right)
$$

is not a lattice variable and nonzero with positive probability. For integral expressions expressing $K_{+}(\cdot)$ and $K_{-}(\cdot)$ as functionals of $\gamma(\cdot)$, see [14], p. 143 and [15].

Actually, the relation (5.6)iii describes the factorisation of

$$
\frac{1-\gamma(\rho)}{(\beta-\alpha) \rho}, \quad \operatorname{Re} \rho=0
$$

as the product of two functions with one the boundary value of a function regular in $\operatorname{Re} \rho<0$, the other regular in $\operatorname{Re} \rho>0$. A further result from $G I / G / 1$ queueing theory is that:

$$
\begin{array}{ll}
\mathrm{E}\left\{\mathrm{e}^{-\rho \mathbf{w}}\right\}=\mathrm{e}^{K_{-}(\rho)}, & \operatorname{Re} \rho \geq 0, \\
\mathrm{E}\left\{\mathrm{e}^{\rho \mathbf{V}}\right\}=\mathrm{e}^{K_{+}(\rho)}, & \operatorname{Re} \rho \leq 0 .
\end{array}
$$

6. The CASE With all $\beta_{j}(\rho)$ Rational

In this section we shall consider the case that all $\beta_{j}(\rho), j=1, \ldots, N$, are rational functions of $\rho$, and no assumptions are made concerning the $\alpha_{j}(\rho), j=1, \ldots, N$.

We start from the relation (3.13) written as: for $j=1,2, \ldots, N$, and $\operatorname{Re} \rho=0$,

$$
\begin{aligned}
& \Phi_{j}(\rho)=\frac{(\beta-\alpha) \rho}{1-\gamma(\rho)}\left[\Psi(\rho) P[I-\Gamma(\rho) P]^{-1}\right]_{j}(1-\gamma(\rho)), \\
& \beta_{j}(\rho)=\frac{b_{1 j}(\rho)}{b_{2 j}(\rho)}, \gamma_{j}(\rho)=\frac{b_{1 j}(\rho)}{b_{2 j}(\rho)} \alpha(-\rho), b_{2}(\rho)=\prod_{j=1}^{N} b_{2 j}(\rho) .
\end{aligned}
$$

By using (5.6)iii we rewrite this relation as: for $j=1, \ldots, N, \operatorname{Re} \rho=0$,

$$
\frac{b_{2}(\rho)}{b_{2 j}(\rho)} \mathrm{e}^{-K_{-}(\rho)} \Phi_{j}(\rho)=\mathrm{e}^{-K_{+}(\rho)} \frac{b_{2}(\rho)}{b_{2 j}(\rho)}\left[\Psi(\rho) P[I-\Gamma(\rho) P]^{-1}\right]_{j}(1-\gamma(\rho)) .
$$

From (3.10), (3.15) and (5.6)i it is seen that the function in the lefthand side of (6.2) is the boundary 
value of a regular function in $\operatorname{Re} \rho>0$ which for $|\rho| \rightarrow \infty,|\arg \rho|<\frac{1}{2} \pi$, behaves as $|\rho|^{\nu-n_{j}}$. By noting that in the elements of the $j$ th column of the matrix in (3.12) the factor $\gamma_{j}(\rho)$ does not occur it is readily seen from (3.15) and (5.6)ii that the righthand side of (6.2) is the boundary value of a function regular in $\operatorname{Re} \rho<0$ and that for $|\rho| \rightarrow \infty, \frac{1}{2} \pi<\arg \rho<\frac{3}{2} \pi$, it behaves as $|\rho|^{\nu-n_{j}}$. Consequently, these functions for $\operatorname{Re} \rho>0$ and $\operatorname{Re} \rho<0$, respectively are each other's analytic continuation. Hence by applying Liouville's theorem it follows that these functions are both a polynomial say, $P_{j}(\rho)$, of degree $\nu-n_{j}$, i.e. for $j=1, \ldots, N$,

$$
\begin{aligned}
& \Phi_{j}(\rho)=\frac{b_{2 j}(\rho)}{b_{2}(\rho)} \mathrm{e}^{K-(\rho)} P_{j}(\rho), \quad \operatorname{Re} \rho \geq 0, \\
& P_{j}(\rho) \text { a polynomial in } \rho \text { of degree } \nu-n_{j}, \nu=\sum_{j=1}^{N} n_{j} \text { the degree of } b_{2}(\rho) .
\end{aligned}
$$

Substitution of (6.4) into (3.6) leads to: for $j=2, \ldots, N, \operatorname{Re} \rho=0$,

$$
\frac{b_{2 j}(\rho)}{b_{2}(\rho)} \mathrm{e}^{K_{-}(\rho)} P_{j}(\rho)-\frac{b_{2 j-1}(\rho)}{b_{2}(\rho)} \gamma_{j-1}(\rho) \mathrm{e}^{K_{-}(\rho)} P_{j-1}(\rho)=-\rho \Psi_{j-1}(\rho),
$$

or by using (5.6)iii: for $j=2, \ldots, N, \operatorname{Re} \rho=0$,

$$
\left[b_{2 j}(\rho) P_{j}(\rho)-b_{2 j-1}(\rho) \gamma_{j-1}(\rho) P_{j-1}(\rho)\right] \mathrm{e}^{K_{+}(\rho)}=\frac{1-\gamma(\rho)}{(\beta-\alpha) \rho} b_{2}(\rho) \rho \Psi_{j-1}(\rho) .
$$

The function in the lefthand side of $(6.5)$ is the boundary value of a function regular for $\operatorname{Re} \rho<0$, cf. (5.6)ii and (6.4), that in the righthand side is also such a function, cf. (3.9) and note that $\{1-\gamma(\rho)\} b_{2}(\rho) / \rho$ has no poles in $\operatorname{Re} \rho \leq 0$, since all $\beta_{j}(\rho)$ are rational. Hence by analytic continuation (6.5) holds for $\operatorname{Re} \rho \leq 0$. It follows from the continuity of $\Psi_{j-1}(\rho)$ in $\operatorname{Re} \rho \leq 0$ that the zeros of

$$
\frac{1-\gamma(\rho)}{(\beta-\alpha) \rho} b_{2}(\rho) \text { in } \operatorname{Re} \rho \leq 0,
$$

should be zeros of

$$
b_{2 j}(\rho) P_{j}(\rho)-b_{2 j-1}(\rho) \gamma_{j-1}(\rho) P_{j-1}(\rho), \quad j=2, \ldots, N
$$

note that $K_{+}(\rho)$ is continuous in $\operatorname{Re} \rho \leq 0$.

By applying Rouche's theorem it is readily shown, cf. [14], p. 323, that the function in (6.6) has for $\beta-\alpha<0$ exactly $\nu$ zeros $\epsilon_{1}, \ldots \epsilon_{\nu}$, say, in $\operatorname{Re} \rho \leq 0$ and their real parts are all negative. Hence, if all these zeros have multiplicitly one; see remark 6.1 below, then: for $\rho=\epsilon_{k}, k=1, \ldots, \nu$,

$$
\begin{array}{ll}
\text { i. } & b_{2 j}(\rho) P_{j}(\rho)-b_{2 j-1}(\rho) \gamma_{j-1}(\rho) P_{j-1}(\rho)=0, \quad j=2, \ldots, N, \\
\text { ii. } & b_{21}(\rho) P_{1}(\rho)-b_{2 N}(\rho) \gamma_{N}(\rho) P_{N}(\rho)=0 ;
\end{array}
$$

the relation in (6.8)ii follows from the last realtion in (3.6) by using the same arguments as above.

From (3.15)i, (5.6)i and (6.4) we have

$$
\frac{b_{2 j}(0)}{b_{2}(0)} P_{j}(0)=1, \quad j=1, \ldots, N
$$

Because $P_{j}(\rho)$ is a polynomial of degree $\nu-n_{j}$, cf. (6.4), and so has $\nu-n_{j}+1$ coefficients the determination of these polynomials requires the determination of 


$$
\sum_{j=1}^{N}\left(\nu-n_{j}+1\right)=N+(N-1) \nu
$$

unknowns. Because $1-\gamma(\rho)$ is the main determinant of the set of equations (3.6) for the $\Phi_{j}(\rho)$, cf. (3.11), it follows that the relation (6.8)ii depends linearly on those in (6.8)i. So it is seen that the conditions (6.8)i and (6.9) represent $(N-1) \nu+N$ inhomogeneous linear equations for the $N+(N-1) \nu$, cf. (6.10), coefficients of the polynomials $P_{j}(\rho), j=1, \ldots, N$. Because the condition $\beta-\alpha<0$ implies that the $w_{n}$-process has a unique stationary distribution, it follows that there exists a unique $\Phi(\rho)$, satisfying (3.15)i, and consequently, the system of linear, inhomogeneous equations (6.8)i and (6.9) has a unique solution if all zeros $\rho=\epsilon_{k}, k=1, \ldots, \nu$, have multiplicity one.

REMARK 6.1. The sum of the multiplicities of the zeros of the function in (6.9) is always equal to $n$. If such a zero, say $\epsilon_{1}$, has a multiplicity larger than one, then $\epsilon_{1}$ should be also a zero with the same multiplicity of (6.7), and it is readily seen that again a system of linear equations for the coefficients of the $P_{j}(\cdot)$ is obtained which determines these coefficients.

From the above it is seen that the polynomials $P_{j}(\rho), j=1, \ldots, N$, may be considered to have been determined. Hence, for the ultimate determination of the vectors $\Phi(\rho)$ and $\Psi(\rho)$ it remains to determine $K_{+}(\rho)$ and $K_{-}(\rho)$. From the preceding section it follows, cf. (5.7), that exp $K(\rho)$, $\operatorname{Re} \rho \geq 0$ is the Laplace-Stieltjes transform of the stationary distribution of the actual waiting time of a $G I / K_{n} / 1$ queue, since $\beta(\rho)=\prod_{j=1}^{N} \beta_{j}(\rho)$ is a rational function of $\rho$. That transform has been determined in [14], section II.5.10. From the results obtained there it follows that, cf. (II.5.190): for $\operatorname{Re} \rho \geq 0$,

$$
\mathrm{e}^{K-(\rho)}=\frac{b_{2}(\rho)}{b_{2}(0)} \prod_{k=1}^{\nu} \frac{\epsilon_{k}}{\epsilon_{k}-\rho} .
$$

Hence from (6.4), (6.9) and (6.11) it follows that: for $j=1, \ldots, N, \operatorname{Re} \rho \geq 0$,

$$
\Phi_{j}(\rho)=\frac{b_{2 j}(\rho) P_{j}(\rho)}{b_{2 j}(0) P_{j}(0)} \prod_{k=1}^{\nu} \frac{\epsilon_{k}}{\epsilon_{k}-\rho}, \quad \operatorname{Re} \rho \geq 0
$$

if it is assumed that all $\epsilon_{k}$ have multiplicity one. Note that $b_{2 j}(\rho) P_{j}(\rho)$ is a polynomial of degree $\nu$.

So we have reached the following result.

For $\beta-\alpha<0$ the Laplace-Stieltjes transforms $\Phi_{j}(\rho), j=1, \ldots, N ; \operatorname{Re} \rho \geq 0$, of the stationary distributions of the actual waiting times $\mathbf{w}_{j}, j=1, \ldots, N$ (in an arrival-cycle) are given by

i. $\quad \Phi_{j}(\rho)=\frac{b_{2 j}(\rho) P_{j}(\rho)}{b_{2 j}(0) P_{j}(0)} \prod_{k=1}^{\nu} \frac{\epsilon_{k}}{\epsilon_{k}-\rho}, \quad j=1, \ldots, N ; \quad \operatorname{Re} \rho \geq 0$

with

ii. $\epsilon_{k}, k=1, \ldots, \nu$, the zeros of

$$
1-\prod_{j=1}^{N} \frac{b_{1 j}(\rho)}{b_{2 j}(\rho)} \alpha_{j}(-\rho) \text { in } \operatorname{Re} \rho<0
$$

iii. $P_{j}(\rho), j=1, \ldots, N$, polynomials of degree $\nu-n_{j}$, determined by the conditions

$$
b_{2 j}(0) P_{j}(0)=b_{2}(0), \quad j=1, \ldots, N,
$$

and 
for $\rho=\epsilon_{k}, k=1, \ldots, \nu$,

$$
\begin{aligned}
& b_{21}(\rho) P_{1}(\rho)-\gamma_{N}(\rho) b_{2 N}(\rho) P_{N}(\rho)=0, \\
& b_{2 j}(\rho) P_{j}(\rho)-\gamma_{j-1}(\rho) b_{2 j-1}(\rho) P_{j-1}(\rho)=0, \quad j=2, \ldots, N .
\end{aligned}
$$

7. The Case With all $\alpha_{j}(\rho)$ Rational

In this section we consider the case that all $\alpha_{j}(\rho), j=1, \ldots, N$, are rational functions of $\rho$, and no assumptions will be made concerning the $\beta_{j}(\rho), j=1, \ldots, N$.

We start from the relations (3.6) and by using (2.9) we have, cf. $(3.8)$ : for $\operatorname{Re} \rho=0, j=2, \ldots, N$.

$$
\begin{array}{ll}
\text { i) } & a_{2 j-1}(-\rho) \Phi_{j}(\rho)-a_{2 j-1}(-\rho) \gamma_{j-1}(\rho) \Phi_{j-1}(\rho)=(\beta-\alpha) \rho \Psi_{j-1}(\rho) a_{2 j-1}(-\rho), \\
\text { ii) } & a_{2 N}(-\rho) \Phi_{1}(\rho)-a_{2 N}(-\rho) \gamma_{N}(\rho) \Phi_{n}(\rho)=(\beta-\alpha) \rho \Psi_{N}(\rho) a_{2 N}(-\rho) .
\end{array}
$$

From (2.10) and (3.15) it is seen that the lefthand side of (7.1)i is the boundary value of a function regular in $\operatorname{Re} \rho>0$, continuous in $\operatorname{Re} \rho \geq 0$, which for $|\rho| \rightarrow \infty,|\arg \rho| \leq \frac{1}{2} \pi$ behaves as $|\rho|^{m_{s-1}}$. Similarly, it is seen that the righthand side in (7.1)i is the boundary value of a function regular in $\operatorname{Re} \rho<0$, continuous in $\operatorname{Re} \rho \leq 0$ and which behaves as $|\rho|^{m_{j-1}}$ for $|\rho| \rightarrow \infty, \frac{1}{2} \pi \leq \arg \rho \leq \frac{3}{2} \pi$. Hence these functions for $\operatorname{Re} \rho<0$ and $\operatorname{Re} \rho>0$ are each other's analytic continuation, and so by applying Liouville's theorem it follows that : for $\operatorname{Re} \rho \leq 0, j=1, \ldots, N$,

$$
\begin{aligned}
& (\beta-\alpha) \rho a_{2 j}(-\rho) \Psi_{j}(\rho)=Q_{j}(\rho), \\
& Q_{j}(\rho) \text { a polynomial in } \rho \text { of degree } m_{j}, Q_{j}(0)=0, \text { cf. (3.8.) }
\end{aligned}
$$

For the present case we have, cf. (3.10), (3.11), (5.6)iii: for $\operatorname{Re} \rho=0$,

$$
\frac{D_{N}(\rho)}{(\beta-\alpha) \rho}=\frac{1-\gamma(\rho)}{(\beta-\alpha) \rho}=\frac{a_{2}(-\rho)-a_{2}(-\rho) \gamma(\rho)}{(\beta-\alpha) \rho a_{2}(-\rho)}=\mathrm{e}^{K_{+}(\rho)-K_{-}(\rho)} .
$$

Because $\beta-\alpha<0$ it follows readily, cf. [14], p. 330, that for the present case $D_{N}(\rho)$ has $\mu$ zeros $\delta_{k}, k=1, \ldots, \mu$, in $\operatorname{Re} \rho \geq 0$, and $\delta_{1}=0, \operatorname{Re} \delta_{k}>0, k=2, \ldots, \mu$, a result easily obtained by applying Rouché's theorem.

REMARK 7.1. For the sake of simplicity it is again assumed that these zeros all have multiplicity one; see also remark 6.1 .

From [14] p. 330, and from section 5 we obtain:

$$
\begin{array}{ll}
\mathrm{e}^{K+(\rho)}=\frac{a_{2}(0)}{a_{2}(-\rho)} \prod_{k=2}^{\mu} \frac{\delta_{k}-\rho}{\delta_{k}}, & \operatorname{Re} \rho \leq 0, \\
\mathrm{e}^{K-(\rho)}=\frac{-a_{2}(0)(\alpha-\beta) \rho}{a_{2}(-\rho)-a_{1}(-\rho) b(\rho)} \prod_{k=2}^{\mu} \frac{\delta_{k}-\rho}{\delta_{k}}, & \operatorname{Re} \rho \geq 0 .
\end{array}
$$

For $\operatorname{Re} \rho=0$, we have, cf. (3.13),

$$
\Phi(\rho)=\frac{(\beta-\alpha) \rho}{1-\gamma(\rho)} \Psi(\rho) D_{n}(\rho) P[I-\Gamma(\rho) P]^{-1},
$$

and so from (7.3): for $\operatorname{Re} \rho=0$, 


$$
\Phi(\rho) \mathrm{e}^{-K_{-}(\rho)}=\frac{\mathrm{e}^{-K_{+}(\rho)}}{a_{2}(-\rho)} \Psi(\rho) a_{2}(-\rho) D_{N}(\rho) P[I-\Gamma(\rho) P]^{-1} .
$$

Hence by using (7.2) and (7.3) we have: for $\operatorname{Re} \rho=0, j=1, \ldots, N$,

$$
\Phi_{j}(\rho) \mathrm{e}^{-K_{-}(\rho)}=\frac{\prod_{k=2}^{m} \delta_{k}}{a_{2}(0)} \frac{a_{2}(\rho)}{\prod_{k=2}^{m}\left(\delta_{k}-\rho\right)} \sum_{i=1}^{N} \frac{Q_{i}(\rho)}{\alpha_{2 i}(\rho)} \frac{D_{N}(\rho)}{(\beta-\alpha) \rho}\left[P[I-\Gamma(\rho) P]^{-1}\right]_{i j} .
$$

From the structure of the matrix in (3.12) and from the fact that the factor $a_{2}(-\rho) / a_{2 i}(-\rho)$ occurs in the righthand side it is seen that this righthand side has a meromorphic continuation in $\operatorname{Re} \rho>0$, since $Q_{i}(\rho)$ is a polynomial. The lefthand side of (7.6) has an analytic continuation in $\operatorname{Re} \rho>0$, see (3.15)i and (5.6)i. Because these continuations are unique it follows that: for $j=1, \ldots, N$,

$$
\sum_{i=1}^{N} \frac{Q_{i}(\rho)}{\alpha_{2 i}(-\rho)} \frac{D_{n}(\rho)}{(\beta-\alpha) \rho}\left[P[I-\Gamma(\rho) P]^{-1}\right]_{i j}=0 \text { for } \rho=\delta_{k}, \quad k=2, \ldots, \mu
$$

note that these $\delta_{k}, k=2, \ldots, \mu$, are the only poles of the mentioned meromorphic continuation.

Next we multiply $(7.1)$ by $\mathrm{e}^{-K_{-}(\rho)}$. Then by using $(7.2),(7.3)$ and $(7.4)$ we obtain: for $\operatorname{Re} \rho=0$, $j=2, \ldots, N$,

$$
\begin{aligned}
& \text { i. } \Phi_{j}(\rho) \mathrm{e}^{-K-(\rho)=} \frac{1}{\alpha_{2 j-1}(-\rho)}\left[\alpha_{2 j-1}(-\rho) \gamma_{j-1}(\rho) \Phi_{j-1}(\rho) \mathrm{e}^{-K_{-}(\rho)}+\right. \\
& \text { ii. } \left.Q_{j-1}(\rho) \frac{a_{2}(-\rho)}{a_{2}(0)} D_{N}(\rho) \prod_{k=2}^{\mu} \frac{\delta_{k}}{\delta_{k}-\rho}\right], \\
& \Phi_{1}(\rho) \mathrm{e}^{-K_{-}(\rho)}=\frac{1}{\alpha_{2 N}(-\rho)}\left[\alpha_{2 N}(-\rho) \gamma_{N}(\rho) \Phi_{N}(\rho) \mathrm{e}^{-K_{-}(\rho)}+Q_{N}(\rho) \frac{a_{2}(-\rho)}{a_{2}(0)} D_{N}(\rho) \prod_{k=2}^{\mu} \frac{\delta_{k}}{\delta_{k}-\rho}\right]
\end{aligned}
$$

note that for $\rho=0$ the relations (7.8) imply that

$$
\Phi_{1}(0)=\Phi_{2}(0)=\ldots=\Phi_{N}(0) .
$$

To determine the polynomials $Q_{j}(\rho), j=1, \ldots, N$, note that $Q_{j}(\rho)$ contains $m_{j}$ coefficients because $Q_{j}(0)=0$, cf. (7.2); so in total $\mu=m_{1}+\ldots+m_{N}$ coefficients have to be determined. Now consider (7.7) for $j=1$, then these conditions lead to $\mu-1$ equations for the $\mu$ unknown coefficients in the polynomials $Q_{j}, j=1, \ldots, N$. Hence together with the norming condition $\Phi_{1}(0)=1$, i.e. by using (7.6) for $j=1$ and $\rho=0$, we obtain a system of $\mu$ linear inhomogeneous equations for the $\mu$ unknowns. Assume for the present that this system possesses a unique solution. Then $\Phi_{1}(\rho)$, $\operatorname{Re} \rho \leq 0$, is determined by (7.6). By taking successively $j=2, \ldots, N$ in (7.8)i explicit expressions for $\Phi_{j}(\rho), \operatorname{Re} \rho=0$, are obtained. It also follows from (7.8)i, or equivalently from (7.1)i and (7.2), that for the expressions so obtained $\alpha_{2 j-1}(-\rho) \Phi_{j}(\rho), \operatorname{Re} \rho>0, j=2, \ldots, N$, has a unique analytic continuation in $\operatorname{Re} \rho>0$. Consequently, it follows from (7.6) that the relations (7.8)i for $j=2, \ldots, N$, are identically satisfied. Hence, by using (7.6) for $j=2, \ldots, N$, it follows that the expressions for $\Phi_{j}(\rho), j=2, \ldots, N$, as constructed above, are regular for $\operatorname{Re} \rho>0$ (note the structure of the matrix in (3.12)), which implies that the zeros of $\alpha_{2 j-1}(-\rho)$ in $\operatorname{Re} \rho>0$ are zeros of the sum between the square brackets in (7.8)i for every $j=2, \ldots, N$.

REMARK 7.2. The analysis above is based, apart from the assumption introduced above, on the 
relations (7.8)i, for $j=2, \ldots, N$, and on the relation (7.7) for $j=1$. It is readily verified that this set of relations is equivalent with the system (7.1), cf. also (3.10), and also equivalent with (3.13), Hence the solution constructed above satisfies (7.1)ii, and similarly (7.8)ii. Note further, cf. also (7.9), that this solution leads to a unique determination of $\Psi_{j}(\rho), \operatorname{Re} \rho \leq 0, j=1, \ldots, N$, and that this solution possesses the properties (3.15). The verification of this is not difficult but requires some algebras.

To complete our analysis it remains to justify the assumption introduced above concerning the uniqueness of the solution of the linear system of inhomogeneous equations. This justification is obtained as in the previous section. Viz. the condition $\beta-\alpha<0$ implies that the $w_{n}$-process has a unique stationary distribution and so there exits a unique $\Phi(\rho)$ satisfying (3.15)i, and consequently the system of equations (7.1)i for $j=2, \ldots, N$, together with (7.6) for $j=1$, should have a unique solution satisfying (3.15)i, and this is only possible if the system (7.7) for $j=1$, together with $\Phi_{1}(0)=1$ determines the $\mu$ coefficients of the $Q_{j}(\rho), j=1, \ldots, N$, uniquely.

\section{SOME SPECIAL CASES}

The discussions in the two preceding sections show that an effective analysis of the periodic Pollaczek waiting processes is possible for quite general cases. In this section we comment briefly on a number of special cases of this Pollaczek model.

A first variant is the mixed case, i.e.: the $\alpha_{j}(\rho), j \in A$, and the $\beta_{j}(\rho), j \in B$ are rational, here $A$ and $B$ are disjoint set and their union is the set $\{1,2, \ldots, N\}$. The analysis for general $N, A$ and $B$ is possible, but quite intricate for $N>2$. For $N=2$ this case has been discussed in detail in [19].

A second variant concerns the case where the $\tau_{j}=t_{j}=2, \ldots, N$ and the $\sigma_{j}, j=1, \ldots, N-1$, are constants, $\tau_{j}=t_{j}$ and $\sigma_{j}=s_{j}$, so only $\tau_{1}$ and $\sigma_{N}$ are true stochastic variables. Again an explicit solution can be constructed, cf.[19]. Actually, the functional equation to be solved here is a standard Riemann Boundary Value Problem. A special case of this variant is the periodic GI/G/1 queueing model where every first customer of the cycle generates the arrival of a fixed number i.e. $N-1$, of subsequent customers with constant service - and interarrival times, each service time being equal to the next interarrival time. This variant may serve as a simple model for bursty traffic.

A third variant of interest concerns the case with all $\sigma_{j}$ constant, $\sigma_{j}=s_{j}$, say, $j=1, \ldots, N$. Put $s=s_{j}+\ldots+s_{N}$. Consider for this process the embedded process $\boldsymbol{w}_{1}^{(n)}, n=1,2, \ldots$, and compare it with the single server model $\mathrm{D} / \mathrm{G} / 1$ with interarrival times equal to $s$ and service time distribution that of the sum of $\tau_{1}, \ldots, \tau_{N}$. For this $D / G / 1$ process the supply to the workload of the server occurs at the equally spaced arrival moments, where as in the $w_{1}^{(n)}$-process the supply to the workload is distributed, (discretely and unevenly) over each of the intervals of length $s$. Hence the supply of the workload for the $\boldsymbol{w}_{1}^{(n)}$-process is a smoother process than that in the D/G/1 system. Consequently, the workload process in this periodic Pollaczek process is smoother, i.e. its jumps are less pronounced than those of the workload process of the $D / G / 1$ system.

\section{ACKNOWLEDGEMENT}

The author is grateful to S.C. Borst, O.J. Boxma, M.B. Combé and G. Koole for their helpful comments in preparing this manuscript.

\section{REFERENCES}

1. Pollaczek, F., Problémes Stochastique Posés par la Phénomène de Formation d'une Queue d'Attente à un Guichet et par des Phénomènes Apparantés, Gauthier Villars, Paris, 1957.

2. Arjas, E., On the use of a fundamental identity in the theory of semi-Markov chains, Adv. Appl. Prob. 4 (1972) 271-284.

3. Asmussen, S., Thorisson, H., A Markov chain approach to periodic queues, J. Appl. Prob. 24 (1987) $215-225$. 
4. Harrison, J.M., Lemoine, A.J., Limit theorems for periodic queues, J. Appl. Prob. 14 (1977) 566-576.

5. Lemoine, A.J., Waiting time and workload in queues with periodic Poisson input, J. Appl. Prob. 26 (1989) 390-397.

6. Bambos, N., Walrand, J., On queues with periodic input, J. Appl. Prob. 26 (1989) 382-389.

7. ÇINLAR, E., Queues with semi-Markovian arrivals, J. Appl. Prob. 4 (1967) 365-379.

8. Neuts, Matrix-Geometric Solutions in Stochastic Models; an algorithmic approach, John Hopkins University Press. Baltimore, 1981.

9. LOYNEs, R.M., The stability of a queue with nonindependent inter-arrival and service times, Proc. Camb. Phil. Soc. 58 (1962) 499-520.

10. Muskhelishvili, N.I., Singular Integral equations, Noordhoff, Groningen, 1953.

11. Michlin, S.G., Pröszdorf, S., Singuläre Integral-Operatoren, Akademie Verlag, Berlin, 1980.

12. ZabreYKo, P.P., E.O., Integral equations, a reference text, Noordhoff, Intern. Publishers, Groningen, 1975.

13. Miller, H.D., A matrix factorization problem in the theory of random variables defined on a finite Markov chain. Proc. Camb. Phil. Soc. 58 (1962) 268-285.

14. Cohen, J.W., The Single Server Queue, North-Holland Publ. Comp., Amsterdam, 1982, 2nd edition

15. Cohen, J.W., Complex functions in Queueing Theory, Pollaczek Memorial Volume, Arch. Elektr. Uebertragung, 47 (1993) 300-310.

16. Lukacs, E., Characteristic Functions, Griffin, London, 1960.

17. Lucantoni, D.M., The BMAP/G/1 queue: a tutorial, Performance Evaluation of Computer and Communication Systems, ed. L. Donatiello \& R. Nelson, Lect. Notes Comp. Sc. SpringerVerlag, Berlin, 1993.

18. Cohen, J.W., Boxma, O.J., Boundary Value Problems in Queueing System Analysis, North Holland Publ. Co, Amsterdam, 1983.

19. Cohen, J.W., On periodic Pollaczek waiting processes, report BS-R9407, Dept. Op. Res., Stat., Syst. Theory, Inst. Math., Comp. Sc., C.W.I., Amsterdam, Januari, 1994. 